\title{
A REACTIVE SCHEDULING APPROACH BASED ON FUZZY INFERENCE FOR HYBRID FLOWSHOP SYSTEMS
}

\author{
Uzun Araz, O.; Eski, O. \& Araz, C. \\ Manisa Celal Bayar University, Industrial Engineering Department, Manisa, Turkey \\ E-Mail: ozlem.araz@cbu.edu.tr, ozgur.eski@cbu.edu.tr, ceyhun.araz@cbu.edu.tr
}

\begin{abstract}
Hybrid flowshops consist of multiple production stages each of which has multiple parallel machines. Scheduling of hybrid flowshops is a NP-hard even in its simplest form. The presence of uncertainty in real-world problems forces the decision makers to reconsider their scheduling decisions in reactive manner. In this study, we proposed a proactive-reactive scheduling approach which allows to be changed dispatching rule set applied in time. The methodology consists of three parts: Shop Floor Management system with a triggering mechanism based on fuzzy inference system, performance prediction of the alternative dispatching rule sets based on Taguchi design, simulation, artificial neural networks, and a multi-criteria decision making methodology for determining new scheduling dispatching rule set. The proposed approach is applied on a real world problem from literature and the results are compared with static approach.

(Received in May 2018, accepted in October 2018. This paper was with the authors 2 months for 1 revision.)
\end{abstract}

Key Words: Hybrid Flowshop, Real-Time Scheduling, Fuzzy Inference System, Simulation

\section{INTRODUCTION}

Flowshops (FS) are manufacturing environments that consists multi stages with one machine in each stage. Hybrid flowshop (HFS) is an extension of FS. Differently from traditional FSs, each production stage may have multiple parallel identical machines in hybrid flowshops. The reasons behind machine duplication in parallel are to increase throughput and the capacity of the shop floor, to balance the production stages, or to reduce the impact of bottleneck machines [1]. HFSs are common manufacturing environment that can be found in different industries such as automotive, chemical, electronic, chemical etc. Scheduling of HFSs is a complex combinatorial problem. Gupta [2] proved that the HFS scheduling problem is NPhard even if the flow line consists of two stages and there is only one machine in the first stage and there are two machines on the second stage.

Scheduling approaches under uncertainty can be categorized as predictive scheduling, completely reactive scheduling, predictive-reactive scheduling, proactive scheduling, and proactive-reactive scheduling [3-5]. In predictive scheduling, schedules that can be applied under all circumstances are created. Completely reactive schedules try to create schedules for immediate future considering the local parameters in real time. In predictive-reactive scheduling, predictive schedules are created for current production environment and schedules can be changed after the occurrence of some certain events such as machine breakdowns, new job arrivals, rush orders, customer demands change, etc. [6-9] or after the performance measures reach their threshold values. Proactive schedules try to generate initial schedules considering the possible future disruptions and apply it for scheduling period without rescheduling. In proactive-reactive approach, the initial schedule is created in a proactive manner then the disruptions are handled by re-scheduling.

As stated in the surveys of [3-5], [10,11], a significant number of studies performed in classical HFS literature which considers static case. However, most solution approaches developed for static case are not appropriate for real world problems. In the most of the real world examples, a production schedule created for a period of time cannot be executed as 
planned. Unexpected events such as machine breakdowns, arrivals of high priority orders, demand changes etc. affect the performance of the schedules.

There have been a limited number of studies for coping with the uncertainties in the HFS environment. Most of these studies are focused on predictive scheduling approaches. Bang and Kim [12] proposed a heuristic in which schedules are created considering bottleneck machines. They also presented a rolling horizon method to cope with the dynamic lot arrivals. Joo et al. [13] considered a three-stage dynamic flexible flowshop scheduling problem in which jobs of multiple types arrive dynamically over time, a quality feedback mechanism is present, and the setup timing and the process defect rate are closely related. Kianfar et al. [14] studied a flexible flowshop system considering dynamic arrival of jobs and the ability of acceptance and rejection of new jobs. Kia et al. [15] performed a simulation based experimental study for scheduling HFSs considering sequence dependent setup times. They concluded that, one of the proposed heuristics performs better for the mean tardiness objective. Lodree et al. [16] proposed a new dispatching rule for minimizing the number of tardy jobs in a dynamic flowshop consisting of $m$ machines in which jobs randomly arrive to the system. Gholami et al. [17] considered the hybrid flowshop scheduling problems in which there are sequence-dependent setup times and machines which suffer stochastic breakdown to optimize objectives based on expected makespan. Kuo et al. [18] developed a robust solution methodology for a dispatching decision for different operating scenarios in hybrid flowshops. In their methodology simulation models of a flowshop with multiple processors is proposed then two multi criteria decision-making methods in combination with Taguchi orthogonal array are used to find the most suitable dispatching rule for every workstation.

There have been a very limited number of HFS studies that uses reactive and proactive approaches considering uncertainty. Lee [19] considered a problem of estimating order flow times in two-stage hybrid flowshops, where orders arrive dynamically and various scheduling schemes can be used. Lee proposed several order arrival estimation models for different scheduling schemes, used in the shop, and considered the current workload and also the expected workload in the near future. Lee conducted simulation experiments in order to show the effectiveness of the proposed methodology. Choi et al. [20] proposed a real-time scheduling mechanism with a decision tree when selecting appropriate dispatching rules in a re-entrant hybrid flowshops. Their scheduling framework consists of real time controller which monitors the system states in real time manner, a scheduler mechanism determines the point of time when a new schedule is to be selected, and a decision tree based rule selector that selects a new dispatching rule without performing time consuming simulation runs. Aufenanger et al. [5] developed an adaptive scheduling methodology that integrates simulation and artificial intelligence techniques in order to schedule a hybrid flowshop under uncertainty. They used artificial intelligence in order to imitate the human process of decision making. Simulation models were used to gather information prior to the production process.

Even though these recent studies have been devoted to scheduling of HFS systems, most of them concentrated on the different parts of the problem such as static scheduling of HFS, dynamic scheduling of HFS, the use of artificial neural network (ANN) for meta-modelling, etc. An effective methodology that can integrate various modelling tools to deal with the multi objective dynamic nature of the HFS is undoubtedly desired. Motivated by above remarks, in this research, we proposed a novel proactive-reactive scheduling approach which integrates fuzzy inference system (FIS), simulation, neural networks and a multi-criteria decision making technique (MCDM) VIKOR. Consequently, the novelty of the work reported in this paper can be summarized as follows; $i$ ) First time the use of FIS as a scheduler mechanism for HFS which determines the point of time when a new dispatching rule set is to be selected; $i$ ) First time the use of VIKOR to select the dispatching rule set which has the best compromise performance according to the selected multiple criteria; iii) First time 
Taguchi design is integrated with ANN and simulation for the scheduling of HFS; iv) The investigation of importance of varying load level of HFS on the performance of reactive scheduling approaches is done first time in the literature.

The rest of the paper is organized as follows. A brief description about ANN, FIS and VIKOR is given in section two. Section three is devoted to proposed methodology. The proposed methodology is applied on a case problem in section four. The final section includes concluding remarks.

\section{METHODS}

In this research, we presented a proactive-reactive scheduling approach which integrates FIS, simulation, ANN and a MCDM technique VIKOR for solving the problem of HFS rescheduling under uncertainty. A brief descriptions about the ANN, FIS and VIKOR which take place in our methodology is given in the following subsections.

\subsection{Artificial neural network modelling}

ANN is a computing architecture that takes its roots from the biological nervous system. An ANN is formed from multiple artificial neurons (processing elements), connected with weights. The input signals are multiplied by the connection weights are first summed then passed through a transfer function in order to produce the output for that neuron.

ANNs have a number of features that offer some useful properties. First, the computation speed of ANNs is very high as a result of parallel computing. Second, ANNs have the ability of learning by adopt their synaptic weights to changes in the behaviour of the surrounding system. Third, ANNs eliminate the need to develop explicit model of a given process, and finally ANNs can represent both linear and non-linear relationships.

A meta-model is a mathematical approximation of a simulation model that represents the relationships between inputs and outputs. The use of simulation may become time consuming due to combinatorial nature of the most of the real world problems. The use of meta-models may overcome this limitation of the simulation. In BP based ANN meta-modelling, simulation output is estimated with a BP neural network whose training set is provided by simulation model [21]. There are several studies in the literature showed that the ANN metamodels can be effectively used for estimation of the system performance measures [21-23].

\subsection{Fuzzy inference system}

Fuzzy inference system (FIS) maps an input space to an output space using fuzzy set theory. In general, FIS consists of 4 modules: i) Fuzzification module: In this module, the crisp inputs are transformed into fuzzy inputs by using fuzzification interface. ii) Knowledge base module: Knowledge base contains the IF-THEN rule sets and fuzzy membership functions based upon the knowledge and understanding of the system. iii) Inference Engine: The inference mechanism applies human reasoning process to compute fuzzy outputs. iv) Defuzzification module: This module transforms the fuzzy outputs obtained from inference module into crisp values. When decision maker's opinion on the performance measures is either linguistic or fuzzy, a FIS allows use of linguistic terms in the form of if-then rules which are more suitable, understandable and applicable for making decisions about the shop floor activities such as changing worker with rules, dispatching rules etc. [24].

There are two common inference methods as Mamdani method [25] and Sugeno method [26]. Although these two methods are quite similar, the output membership functions are either linear or constant in Sugeno method whereas the output membership functions are expected to be fuzzy sets in Mamdani method. Comparing with Mamdani method, Sugeno's 
method is computationally efficient, it also works well with optimization and adaptive techniques and it is convenient to mathematical analysis. Sugeno method is well suited for dynamic, non-linear systems [27].

\subsection{VIKOR}

VIKOR is a multiple criteria decision making technique which was introduced by Opricovic [28]. VIKOR method is appropriate for the situations where decision makers are not able to express his/her preferences at the initial stages of the system design [28]. This method determines a compromise solution which is closest to the ideal for the problems with conflicting criteria. In VIKOR method, the compromise ranking is done by comparing the measure of closeness to the ideal alternative under the assumption of each alternative is evaluated according to each criterion function. The various $J$ alternatives are denoted as $a_{1}$, $a_{2}, \ldots, a_{j}$. For alternative $a_{j}$, the related rating of the $i^{\text {th }}$ aspect is denoted by $f_{i j}$ which is the value of $i^{\text {th }}$ criterion function for the alternative $a_{j} ; n$ is the number of criteria.

The compromise solution $F^{c}$ is a feasible solution that is the "closest" to the ideal $F^{*}$, and compromise means an agreement established by mutual concessions $\Delta f_{1}=f_{1}^{*}-f_{1}^{c}$ and $\Delta f_{2}=f_{2}^{*}-f_{2}^{c}$. Development of the VIKOR method started with the following form of $L p$ metric [29] which represents the distance function called as the group regret for a decision:

$$
L_{p j}=\left\{\sum_{i=1}^{n}\left[\frac{w_{i}\left(f_{i}^{*}-f_{i j}\right)}{f_{i}^{*}-f_{i}^{-}}\right]^{p}\right\}^{\frac{1}{p}} \quad 1 \leq p \leq \infty ; j=1,2, \ldots, J
$$

The compromise ranking algorithm VIKOR has the following steps [28-30].

Step 1: Determine the best $f_{i}^{*}$ and the worst $f_{i}^{-}$values of all criterion functions, $i=1,2, \ldots, n$. If the $i^{\text {th }}$ function represents a benefit then:

$$
f_{i}^{*}=\max _{j} f_{i j} \quad f_{i}^{-}=\min _{j} f_{i j}
$$

If the $i^{\text {th }}$ function represents a cost then:

$$
f_{i}^{*}=\min _{j} f_{i j} \quad f_{i}^{-}=\max _{j} f_{i j}
$$

Step 2: Compute the values $S_{j}$ and $R_{j}, j=1,2, \ldots, J$, by relations:

$$
\begin{aligned}
S_{j} & =\sum_{i=1}^{n} w_{i}\left(f_{i}^{*}-f_{i j}\right) /\left(f_{i}^{*}-f_{i}^{-}\right) \\
R_{j} & =\max _{i}\left[w_{i}\left(f_{i}^{*}-f_{i j}\right) /\left(f_{i}^{*}-f_{i}^{-}\right)\right]
\end{aligned}
$$

where $w_{i}$ are the weights of criteria, expressing their relative importance.

Step 3: Compute the values $Q_{j}, j=1,2, \ldots, J$, according to the $S_{j}$ and $R_{j}$

$$
\begin{gathered}
Q_{j}=v *\left(S_{j}-S^{*}\right) /\left(S^{-}-S^{*}\right)+(1-v)\left(R_{j}-R^{*}\right) /\left(R^{-}-R^{*}\right) \\
S^{*}=\min _{j} S_{j}, S^{-}=\max _{j} S_{j}, R^{*}=\min _{j} R_{j}, R^{-}=\max _{j} R_{j}
\end{gathered}
$$

and $v \in[0,1]$ is the weight of the strategy of the "the majority of criteria" (or "the maximum group utility").

Step 4: Sort the values of $S, R$ and $Q$ in decreasing order and rank the alternatives.

Step 5: Propose as a compromise solution the alternative $\left(a^{\prime}\right)$ which is ranked the best by the measure $Q$ (minimum), if the following two conditions are satisfied:

- Condition 1. The acceptable advantage:

$$
Q\left(a^{\prime \prime}\right)-Q\left(a^{\prime}\right) \geq D Q
$$

where $a^{\prime \prime}$ is the alternative with second position in the ranking list by $Q$; 


$$
D Q=1 /(J-1), J \text { is the number of alternatives }
$$

- Condition 2. The acceptable stability in decision making:

Alternative $a^{\prime}$ must also be the best ranked by $S$ or/and $R$. This compromise solution is stable within a decision making process, which could be: "voting by majority rule" (when $v>0.5$ is needed), or "by consensus" ( $v \approx 0.5)$, or "with veto" $(v<0.5)$. Here, $v$ is the weight of the decision making strategy "the majority of criteria" (or "the maximum group utility'). If one of the conditions is not satisfied, then a set of compromise solutions is proposed, which consists of:

- Alternatives $a^{\prime}$ and $a^{\prime \prime}$ if only condition $C_{2}$ is not satisfied, or

- Alternatives $a^{\prime}, a^{\prime \prime}, \ldots, a^{(M)}$ if condition $C_{1}$ is not satisfied; and $a^{(M)}$ is determined by the relation for $Q\left(a^{(M)}\right)-Q\left(a^{\prime}\right)<D Q$ for maximum $M$ (the positions of these alternatives are "in closeness").

\section{PROPOSED METHODOLOGY}

The proposed methodology deals with proactive-reactive scheduling of hybrid flowshop systems which allows to be changed dispatching rule set applied in time. The methodology consists of three main parts: Shop Floor Management system with a triggering mechanism based on fuzzy inference system (FIS), performance prediction of the alternative dispatching rule sets based on Taguchi design, simulation and ANN, multi-criteria decision making methodology for determining new scheduling dispatching rule set. Fig. 1 depicts the relations and data flow between parts of the proposed methodology.

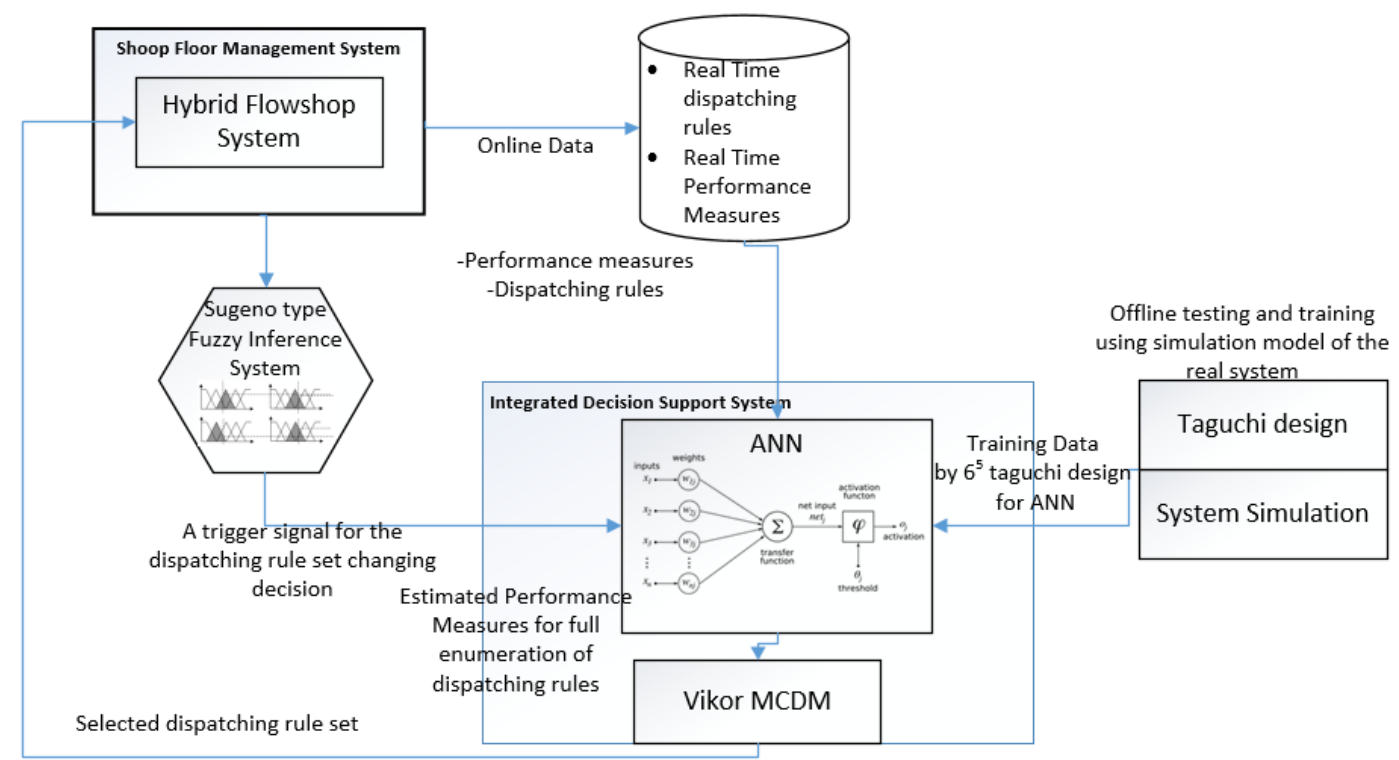

Figure 1: Architecture of the proposed methodology.

The aim of this methodology is to design a reactive scheduling approach in which the rescheduling decisions is made considering the system state variables and the preferences of the decision makers. When a disruption occurs and rescheduling decision is made, then the new scheduling rules that will be applied for the new circumstance are determined. The proposed methodology consists of two main parts as offline stage and online stage.

\subsection{Offline stage}

The aim of the studies performed in offline stage is to create models that will be used in online stage. These studies consist of 4 main modelling studies: 
- Simulation modelling: Real world HFS problems are complex problems that have stochastic and dynamic nature. Simulation is an effective tool for analysing such systems. At this stage, the simulation models of the real system were built and analysed in order to set the system parameters such as arrival rate, processing times and define the performance measures to be considered.

- ANN modelling: In proposed methodology when a rescheduling decision is made, instead of running simulation models, the system performance measures for different rule sets are estimated by ANN based meta-models.

- Taguchi design: A trained ANN model can represent the relationship between parameters and output performance measures. However, training process may require wide range of training data for most of the real world problems. The number of training data and the selection procedure affect the precision of the solutions. In such cases, experimental design techniques are generally applied to reduce the number of the experiments needed. Hence, we used Taguchi method [31] which is one of the fractional designs in order to obtain more accurate results using smaller training data set. Taguchi Orthogonal Array design is a type of general fractional factorial design. It allows us to consider a selected subset of combinations of multiple factors at multiple levels. An orthogonal array of the $s$ elements is denoted by $L_{N}\left(s^{m}\right)$ which can be viewed as a subset of the possible $s^{m}$ test runs of a complete factorial plan in $m$ factors each having $s$ levels. The number of experiments $(N)$ in fractional design can be denoted by [32]:

$$
N=(s-1) \cdot m+1
$$

- VIKOR: The performance of the HFS systems is generally measured by more than one performance measures. The proposed methodology evaluates the alternative solutions at each rescheduling decision points considering the performance measures such as Mean Flow Time, Service Level, Mean Tardiness. In this study, VIKOR ranks the alternative dispatching rule combinations and determines the compromise solution that is closest to the ideal.

\subsection{Online stage}

This stage includes the application of the proposed methodology on the real world HFS system. Online stage consists of three main modules (a, b, c):

\section{a) Shop floor management system (SFMS)}

SFMS collects the data such as performance measures, dispatching rules etc. from hybrid floor shop production system and transmits to the fuzzy inference system.

\section{b) A triggering mechanism based on fuzzy inference system}

This module analyses the data transmitted from SFMS, and computes fuzzy membership function values. This system makes the rescheduling decision considering fuzzy membership functions. As mentioned before, the proactive-reactive scheduling of hybrid flowshop systems allow to change the dispatching rule set applied in time. The timing decision is generally given by periodically or a threshold based triggering procedure. In this paper, a novel fuzzy triggering procedure is also proposed which lies in between the periodically and threshold based procedure. The proposed fuzzy "triggering" procedure is based on Sugeno type FIS and aims to answer the question of when it is necessary to chance the dispatching rule set applied to the HFS system. The Sugeno type FIS considers the performance measures of HFS as input variables such as mean flow time, service level, etc. These variables take three imprecise values: low, medium and high. An example of the membership functions is given in Fig. 2. 


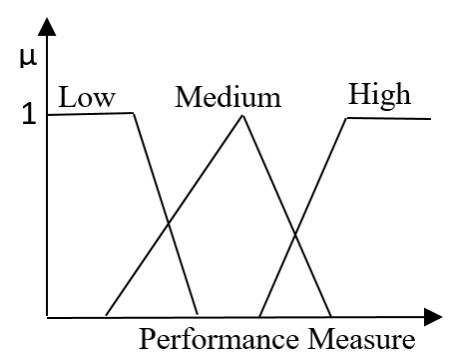

Figure 2: An example of the fuzzy membership functions of performance measures.

The antecedent of Sugeno type FIS involves some linguistic variables and the consequent is a crisp action with associated weights. Therefore, there are two crisp consequences: change dispatching rules $(C)$ or do not change dispatching rules $(D N C)$. Since each of the performance measures has three states, i.e. low, medium and high, the number of the fuzzy rules depends on the number of the number of the performance measures. For instance, fuzzy triggering procedure needs 27 rules in order to make a rescheduling decision for a HFS with three performance measures.

Since Fuzzy triggering procedure uses a zero order Sugeno model, the constant output value $\left(z_{k}\right)$ of making "C" decision is set to one while the constant value $\left(z_{k}\right)$ of making "DNC" decision is set to zero (Fig. 3). The output level of each rule is weighted by the firing strength $w_{k}$ of the rule [33], which can be calculated using "and operator" as follows:

$$
w_{k}=\min \left\{\mu_{1}, \mu_{2}, \ldots, \mu_{K}\right\},
$$

where $\mu_{1}$ to $\mu_{K}$ are the membership functions for $K$ performance measures, respectively.

The final output $(F O)$ is the weighted average of all the outputs of the rules, defined as

$$
F O=\left(\sum_{k=1}^{K}\left(w_{k} z_{k}\right) /\left(\sum_{k=1}^{K} z_{k}\right)\right.
$$

Decision $D$ is then defined as follows:

$$
D=\left\{\begin{array}{llr}
C & \text { if } \quad F O \leq 0.5 \\
D N C & \text { otherwise }
\end{array}\right.
$$

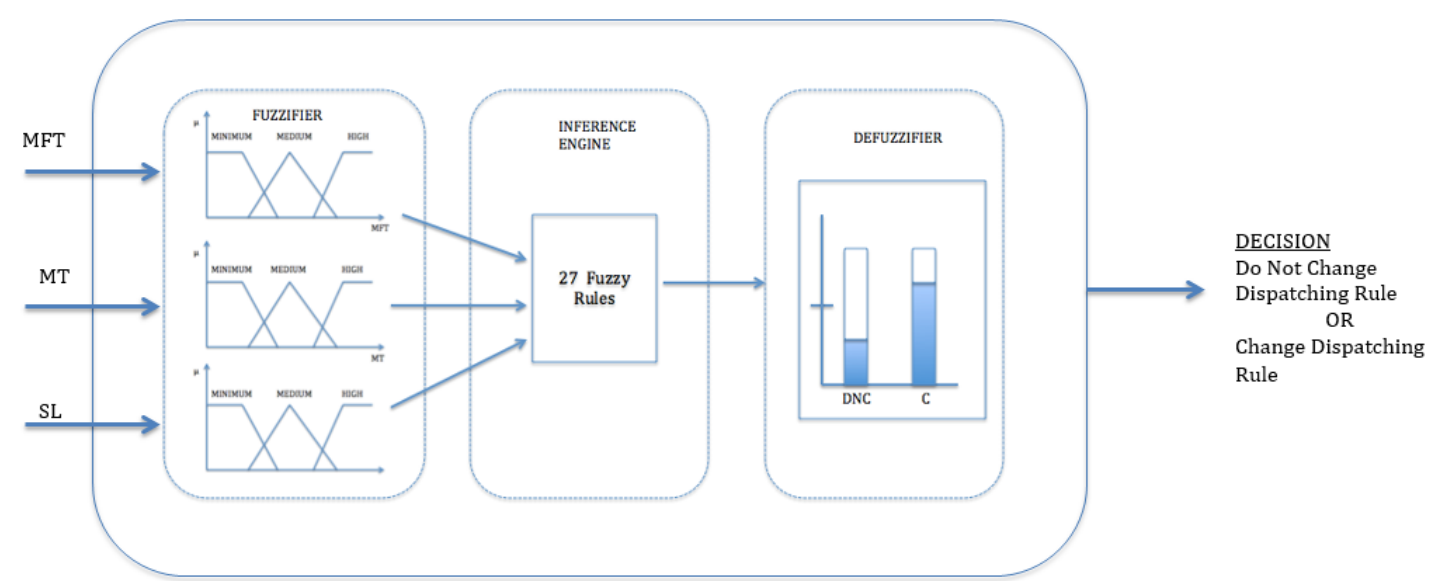

Figure 3: The proposed fuzzy Sugeno Model for fuzzy “triggering” procedure.

The fuzzy controller of the proposed methodology is made of a fuzzification part and an if-then rule part. In the fuzzification part, the decision maker should determine the input variables, linguistics terms of these variables and their membership functions. The fuzzy ifthen rule part uses variables previously defined both in the condition and the conclusion of the rules. Then the defuzzified output is calculated by using Eq. (11). In the proposed methodology, the input variables are defined as the performance values of the hybrid 
flowshop system, the crisp output is defined as a measure of the state which represents that the something is going wrong in the system and the dispatching rule set should be changed with the new one by using Eq. (12).

\section{c) Integrated decision making system}

The proposed "Integrated decision support system" executed after the rescheduling decision is made. This segment consists of two main parts: ANN based meta-modelling and VIKOR. The system state variables and the dispatching rule set applied when rescheduling decision is made and the all possible rule sets that can be applied after the rescheduling point are used as the input data for ANN based meta-model. The performance measures for each alternative are estimated by using the ANN meta-model. The dispatching rule set, which has the best compromise performance, is selected using the compromised ranking method, known as VIKOR, according to the selected evaluation criteria. VIKOR uses values of the performance measures generated form the ANN model. Therefore, the proposed system works offline and fast. VIKOR evaluates and ranks the alternative solutions. The rule set that gives the best result according to VIKOR is employed for the upcoming period. Then, the "system monitoring" module starts to collect real time data again.

\section{NUMERICAL EXAMPLE}

The case problem is similar to the manufacturing system used in [18]. The manufacturing system is composed of 10 production stages. The number of machines in each stage and setup times for each stage are given in Table I. There are 4 different product sizes and 6 material types. Differently from original case, $i$ ) Inter-arrival times are assumed to have an exponential distribution with a mean of $1440 \mathrm{~min}$, ii) Failures are ignored, iii) Order sizes are uniformly distributed with uniform $(5,7)$, iv) Total Work Content (TWK) rule [34] is used in due date assignment, v) FIFO, TIS, SPT, AT-RPT, PT/TIS dispatching rules are used, vi) Average Service Level $(S L)$, Mean Tardiness $(M T)$, Mean Flow Time (MFT) objectives are considered.

Table I: The machine data.

\begin{tabular}{|c|c|c|c|}
\hline No & Stage name & Number of machines & Setup time \\
\hline 1 & Stacking & 17 & Uniform $(27,33)$ \\
\hline 2 & Pressing & 1 & 0 \\
\hline 3 & Cutting & 4 & Uniform $(25,33)$ \\
\hline 4 & Binder Burnout & 16 & 0 \\
\hline 5 & Sintering & 2 & Const $(1440)$ \\
\hline 6 & Tumbling & 8 & 0 \\
\hline 7 & Dipping & 4 & Uniform $(29,35)$ \\
\hline 8 & Curing & 4 & Const $(1440)$ \\
\hline 9 & Plating & 1 & Uniform $(1,2)$ \\
\hline 10 & Testing & 12 & Uniform $(20,29)$ \\
\hline
\end{tabular}

To show the effectiveness of the proposed approach, a simulation model of the case study is constructed using Arena simulation software (Version 14). It is assumed that the dispatching strategy will have an influence on highly loaded workstations [18]. The proposed simulation models are executed for 2000 orders with a warm up period of 10.000 minutes. The variance reduction technique of common random numbers is used for synchronization of random numbers in order to compare alternatives in similar conditions. Ten replications were made for each combination and the SL, MT, and MFT levels were computed. The workstations 3, 5, 6, 8, 9 and 10 are identified as highly loaded workstations. Consequently, highly loaded workstations are considered in this research. 
In the experimental study, five well known dispatching heuristics for job sequencing are selected: FIFO (First in First Out), TIS (Time in System), SPT (Shortest Processing Time), AT-RPT (Arrival Time-Remaining Processing Time), PT-TIS (Process Time-Time in System). In order to highlight the proactive-reactive feature of the proposed approach, the results obtained by the proposed approach are compared with the static case which uses the best dispatching rule set obtained by Taguchi design. In the static case, it is assumed that the initial dispatching rule set is not changed during the simulation run. On the other hand, in the proposed methodology, it is assumed that the initial dispatching rule set is the same with static case, but it is allowed to be changed in some points of the time determined by the fuzzy inference system.

In the first step of the proposed methodology, the triggering mechanism which determines the point of time to change the dispatching rule set is constructed. The linguistic terms and the membership degrees of the performance measures were defined as triangular fuzzy numbers. The rules of the FIS models were developed by considering every combination of input and output parameters. As a result, the FIS model has $27\left(3^{3}\right)$ rules. Two examples of rule base of the FIS model can be shown as follows:

- if $S L$ is Medium and MT is Low and MFT is Medium then "do not changed DPRs",

- if $S L$ is Medium and MT is Low and MFT is High then "Change DPRs".

In the second step, a Taguchi design was conducted to determine the dispatching rules for each workstation in the HFS system for training ANN. Taguchi $\mathrm{L}_{25}$ design based algorithm which is used for generating experimental design points is given below:

BEGIN

Step I: Choose convenient orthogonal array from the Taguchi's standard orthogonal arrays.

Define factor levels for each design point and define intervals for each level.

Determine the number of experiment $(N O E)$ design points from the selected Taguchi's design.

Step II: Define the parameters: $i=1, j=1, k=1$. Set $A_{i}=\left\{r_{i}, i=1,2, \ldots, N\right\}$. Set $R_{k}=\left\{A_{i} \mathrm{U} A_{j}\right\}$.

Step III: Define the design points.

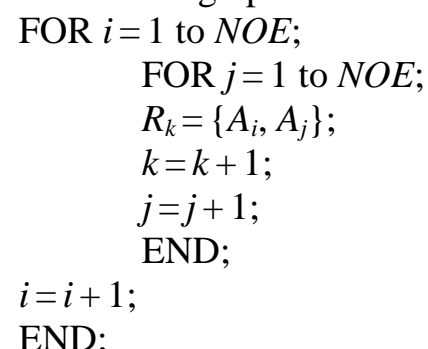

END.

where:

$N$ - number of factors,

$A_{i}$ - set of scheduling rules that will be used for the stations $3,5,6,8,9,10$ at the beginning of the analysis period (1: FIFO, 2: TIS, 3: SPT, 4: AT-RP, 5: PT-TIS),

$A_{j}$ - set of scheduling rules that will be used for the stations $3,5,6,8,9,10$ when rescheduling decision is made,

$R_{k}$ - union of $A_{i}$ and $A_{j}$ for the $k^{\text {th }}$ experiment.

First 6 elements include the scheduling rules that will be applied in 6 workstations at the start of the period and the following 6 elements indicate the scheduling rules that will be applied when a disturbance occurred and rescheduling decision is made. For example: if $A_{1}=\{1,1,1,1,1,1\}$ and $A_{2}=\{1,2,2,2,2,2\}$, then, $R_{1}=\{1,1,1,1,1,1,1,2,2,2,2,2\}$. It means that FIFO dispatching rule will be used for each station at the start of the period. After the rescheduling decision, FIFO dispatching rule will be used for station 3, and TIS dispatching rule will be used for other stations. 
In this experiment, we have 6 factors (number of stations) and each factor has 5 levels (dispatching rules). A full factorial experiment would require a total of $15625^{2}$ experiments $\left(5^{6}=15625\right.$ experiments for time period just before scheduling point and 15625 experiments for forthcoming period). We conducted a Taguchi experiment with $\mathrm{L}_{25}\left(5^{6}\right)$ orthogonal array, which is suitable for 6 factors, 5 levels. Total of $25^{2}=625$ design points were determined by Taguchi method, then the performance values of these experiments were obtained from the simulation model of the system.

At the next step, an ANN model was trained and tested by using the design points determined from Taguchi Design. The ANN model is then used to predict the performance of all dispatching rule combinations. We developed three backpropagation ANN models in order to estimate $M L T, S L T$ and $M T$ performance measures for each alternative. ANN models are executed when rescheduling decision is made and the performance measures of the alternatives are estimated. Each ANN model has 63 input nodes. The first 30 nodes represent the dispatching rules employed at each station (6 stations and 5 dispatching rules) for the time period just before the rescheduling point. The next 3 nodes represent the MLT, SLT and MT performance measures for the time period just before the rescheduling point and the last 30 nodes represent the dispatching rules that will be used for the forthcoming period.

The design parameters such as number of hidden layers, the number of nodes in the hidden layers, the type of transfer function, learning rate, and momentum rate affect the performance of the ANN. In general, these design parameters are determined by trial and error [35]. The design parameters are set by using trial and error method and presented in Table II.

In the proposed methodology, considering the multi-criteria nature of the problem, the best dispatching rule combination is determined by VIKOR method. Finally, the system is simulated during one month. The results are summarized in Table III.

Table II: The parameter sets of ANN models.

\begin{tabular}{|l|c|c|c|}
\hline Parameters & MFT_NN & SL_NN & MT_NN \\
\hline \# of inputs & 63 & 63 & 63 \\
\hline \# of outputs & 1 & 1 & 1 \\
\hline \# of hidden layers & 1 & 1 & 1 \\
\hline \# of neurons in hidden layers & 3 & 3 & 3 \\
\hline Learning rate & 0.1 & 0.1 & 0.1 \\
\hline Momentum rate & 0.7 & 0.7 & 0.7 \\
\hline Activation type & sigmoid & sigmoid & sigmoid \\
\hline$M S E$ & 0.0012074 & 0.0011979 & 0.0034800 \\
\hline
\end{tabular}

In Fig. 4, the performance of static dispatching rules and the proposed approach are compared in terms of three performance criteria. The results are averaged over 10 independently generated test instances for each case. As it can be seen from the results in Fig. 4, the proposed approach outperformed static dispatching rule sets in all performance measures. The mean MFT and MT values of the proposed methodology are lower than those of the static case on all 16 time points, and the mean of $S L$ values are also better than those of the static case.

Manufacturing systems are generally operated at varying load levels which can be controlled in a simulation model by changing the inter-arrival times of demand and due date tightness. In order to examine the efficiency of the proposed methodology for different load levels, nine scenarios are created by changing the inter-arrival times of demand and due date tightness. The three levels used for inter-arrival times of demand and due date tightness in this study are \{high, medium, low $\}$ and \{tight, medium, large\}, respectively. The proposed methodology is applied on these scenarios and performance of the proposed methodology is 
compared with the solutions obtained from static rule sets. In static case, 5-1-5-4-3-2 rule combination, which is the best combination for period 1, is used for all other periods.

Table III: Results of the proposed methodology.

\begin{tabular}{|c|c|c|c|c|c|c|c|c|c|}
\hline \multirow{3}{*}{ Period } & \multirow{3}{*}{$\begin{array}{c}\text { Start time } \\
\text { (minutes) }\end{array}$} & \multirow{3}{*}{$\begin{array}{c}\text { Finish } \\
\text { time } \\
\text { (minutes) }\end{array}$} & \multirow{3}{*}{$\begin{array}{c}\text { Dispatching rules } \\
\text { for work stations } \\
\text { 3-5-6-8-9-10 }\end{array}$} & \multicolumn{6}{|c|}{ Performance measures } \\
\hline & & & & \multicolumn{2}{|c|}{$M F T$} & \multicolumn{2}{|c|}{$S L$} & \multicolumn{2}{|c|}{$M T$} \\
\hline & & & & D & $\mathbf{S}$ & D & $\mathbf{S}$ & D & $\mathbf{S}$ \\
\hline 1 & 0.00 & 377.58 & $5-1-5-4-3-2$ & 337.77 & 337.77 & 0.80 & 0.80 & 80.67 & 80.67 \\
\hline 2 & 377.58 & 434.11 & $3-1-4-2-4-1$ & 367.27 & 388.16 & 0.81 & 0.82 & 101.98 & 125.93 \\
\hline 3 & 434.11 & 463.78 & $2-1-5-3-4-1$ & 394.02 & 441.63 & 0.80 & 0.63 & 125.06 & 166.42 \\
\hline 4 & 463.78 & 479.17 & $1-1-5-3-5-2$ & 392.53 & 454.68 & 0.80 & 0.61 & 122.78 & 166.56 \\
\hline 5 & 479.17 & 494.57 & $2-1-5-2-5-2$ & 391.72 & 455.43 & 0.80 & 0.61 & 121.25 & 163.83 \\
\hline 6 & 494.57 & 510.04 & $1-1-5-3-5-2$ & 390.41 & 469.00 & 0.80 & 0.59 & 119.74 & 167.41 \\
\hline 7 & 510.04 & 525.05 & $1-1-5-2-5-2$ & 398.72 & 487.36 & 0.79 & 0.57 & 125.11 & 168.37 \\
\hline 8 & 525.05 & 545.80 & $2-1-4-2-4-1$ & 401.72 & 499.05 & 0.79 & 0.56 & 127.91 & 172.89 \\
\hline 9 & 545.80 & 566.69 & $2-1-5-3-5-2$ & 410.58 & 506.99 & 0.80 & 0.55 & 136.09 & 190.21 \\
\hline 10 & 566.69 & 587.33 & $2-1-5-2-5-2$ & 425.58 & 498.27 & 0.79 & 0.56 & 150.73 & 192.39 \\
\hline 11 & 587.33 & 607.93 & $1-3-5-5-4-2$ & 443.27 & 515.29 & 0.80 & 0.55 & 169.57 & 217.07 \\
\hline 12 & 607.93 & 629.42 & $5-1-5-1-5-1$ & 444.14 & 516.76 & 0.80 & 0.55 & 170.93 & 215.42 \\
\hline 13 & 629.42 & 650.12 & $4-2-5-2-5-1$ & 446.52 & 503.44 & 0.80 & 0.57 & 173.88 & 217.25 \\
\hline 14 & 650.12 & 670.95 & $2-1-5-2-5-2$ & 442.55 & 507.01 & 0.80 & 0.56 & 169.88 & 211.86 \\
\hline 15 & 670.95 & 691.66 & $1-1-4-3-4-1$ & 438.22 & 514.37 & 0.80 & 0.55 & 165.67 & 209.47 \\
\hline 16 & 691.66 & 712.62 & $2-1-5-2-5-2$ & 447.78 & 523.72 & 0.79 & 0.54 & 172.89 & 208.37 \\
\hline
\end{tabular}

Remark: D - Dynamic; S - Static
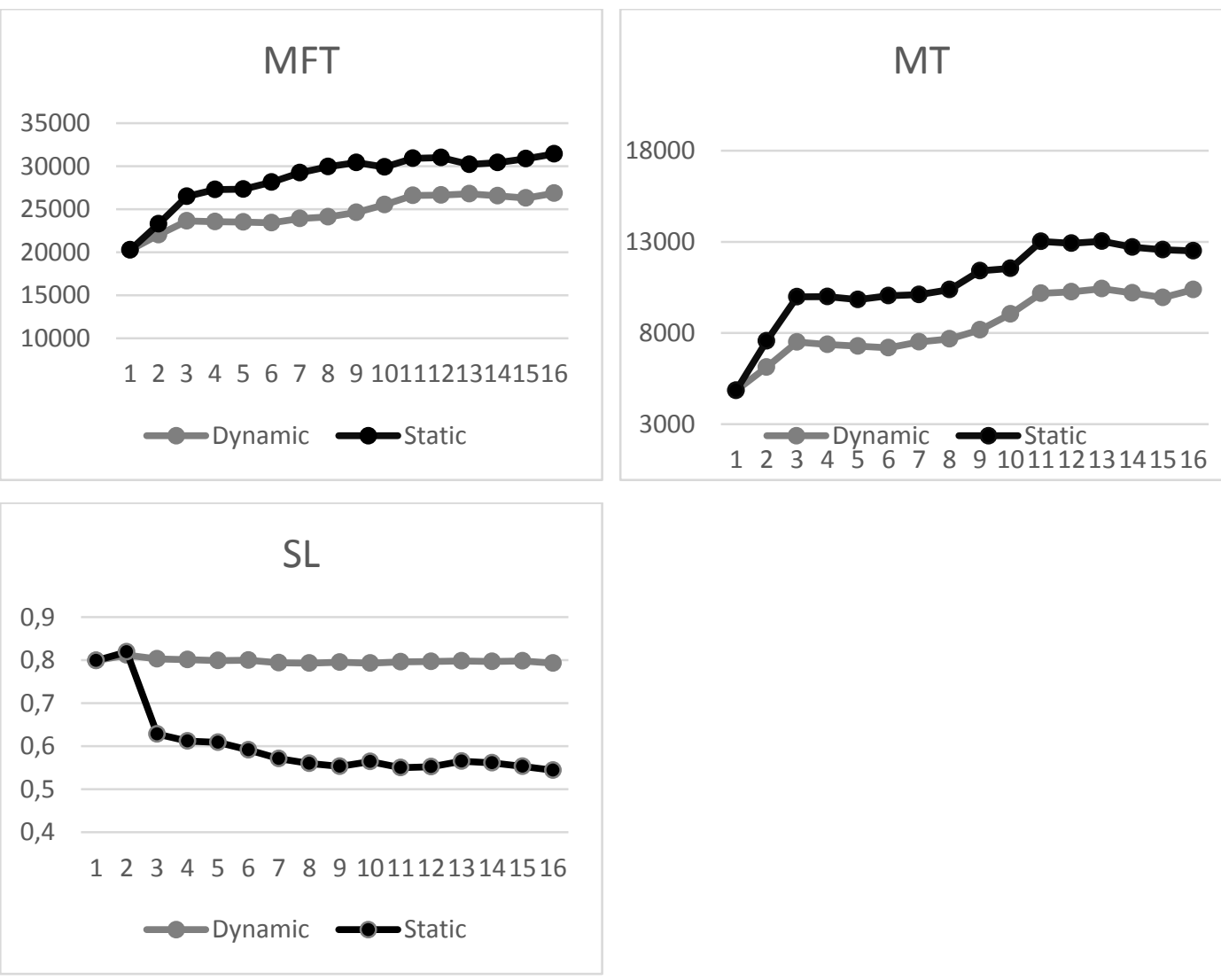

Figure 4: Comparison of the results. 
As it can be seen from Table IV, the proposed methodology has better performance than the static case in terms of all performance measure on all scenarios. When the inter-arrival time is low and due dates are tight, the improvement of the performance measures becomes more obvious. For all other cases, the proposed methodology also outperforms the static case thanks to the proactive-reactive nature of the methodology. It should be noted though that the proposed methodology performs some modifications on dispatching rules applied in time in order to improve the performance measures according to the varying load level of the shop floor.

Table IV: The results of experimental study.

\begin{tabular}{|c|c|c|c|c|c|c|}
\hline \multicolumn{2}{|c|}{ Distribution } & \multicolumn{2}{c|}{ Due date } & \multicolumn{2}{c|}{$\%$ improvement $(\Delta)$} \\
\hline expo $(x)$ & $\begin{array}{c}\text { Inter-arrival } \\
\text { time }\end{array}$ & $\begin{array}{c}k- \\
\text { value }\end{array}$ & $\begin{array}{c}\text { Due date } \\
\text { tightness }\end{array}$ & $M F T$ & $M T$ & $S L$ \\
\hline 1425 & Low & 1 & Tight & 86.7429 & 54.1884 & 47.1199 \\
\hline 1900 & Medium & 1 & Tight & 66.5095 & 40.9961 & 35.9320 \\
\hline 2375 & High & 1 & Tight & 52.8945 & 37.8601 & 28.1982 \\
\hline 1425 & Low & 3 & Medium & 74.6743 & 40.4242 & 43.3831 \\
\hline 1900 & Medium & 3 & Medium & 73.2294 & 36.0018 & 39.4879 \\
\hline 2375 & High & 3 & Medium & 50.2895 & 32.8321 & 21.5000 \\
\hline 1425 & Low & 5 & Large & 28.6743 & 22.3343 & 21.9337 \\
\hline 1900 & Medium & 5 & Large & 16.5095 & 11.4127 & 18.3871 \\
\hline 2375 & High & 5 & Large & 1.2895 & 1.4130 & 3.5450 \\
\hline
\end{tabular}

It can be seen that the improvement of the proposed methodology is not significant only on one case in which the inter-arrival time is high and due dates are large. It is obvious that this scenario relaxes the manufacturing environment with low machine utilization levels. In such cases, the need to change the dispatching rule becomes less important when compared to the other cases. At the low load level scenario with high inter-arrival time and loose due dates the proposed methodology made only a few changes to the initial schedule. This is probably because the production system becomes insensitive to the changes in rule sets when interarrival times are high and large due dates are assigned.

Consequently, we can conclude that the proposed approach outperforms the static case in all instances and the improvements on the results inherently become more significant as the load level of HFS system increases. The results of numerical experiments strongly suggest that the proposed approach can be seen as an alternative for the scheduling of the HFS systems.

\section{CONCLUSIONS}

In this research, we have addressed the problem of HFS rescheduling under uncertainty. For this purpose, we presented a proactive-reactive scheduling approach which integrates FIS, simulation, ANN and a MCDM technique VIKOR. The proposed approach was applied on a real world problem taken from the literature and the results were compared with static approach. The results showed that the proposed approach gives better results in dynamic production environments.

Although the results of proposed methodology are promising, the further research is required in the following directions. First, the effects of machine failures can be examined. Second, the effectiveness of the methodology can be improved by employing a methodology for determining the parameters of the fuzzy inference system. Third, a sensitivity analysis can be performed to show the effectiveness of the methodology for different problem sizes. 


\section{REFERENCES}

[1] Naderi, B.; Gohari, S.; Yazdani, M. (2014). Hybrid flexible flowshop problems: models and solution methods, Applied Mathematical Modelling, Vol. 38, No. 24, 5767-5780, doi:10.1016/j.apm.2014.04.012

[2] Gupta, J. N. D. (1988). Two-stage hybrid flowshop scheduling problem, Journal of Operational Research Society, Vol. 39, No. 4, 359-364, doi:10.1057/jors.1988.63

[3] Aytug, H.; Lawley, M. A.; McKay, K.; Mohan, S.; Uzsoy, R. (2005). Executing production schedules in the face of uncertainties: a review and some future directions, European Journal of Operational Research, Vol. 161, No. 1, 86-110, doi:10.1016/j.ejor.2003.08.027

[4] Sabuncuoglu, I.; Goren, S. (2009). Hedging production schedules against uncertainty in manufacturing environment with a review of robustness and stability research, International Journal of Computer Integrated Manufacturing, Vol. 22, No. 2, 138-157, doi:10.1080/ 09511920802209033

[5] Aufenanger, M.; Varnholt, H.; Dangelmaier, W. (2009). Adaptive flow control in flexible flowshop production systems - a knowledge-based approach, Proceedings of the 2009 Winter Simulation Conference, 2164-2175, doi: $10.1109 /$ WSC.2009.5429735

[6] Swaminathan, R.; Pfund, M. E.; Fowler, J. W.; Mason, S. J.; Keha, A. (2007). Impact of permutation enforcement when minimizing total weighted tardiness in dynamic flowshops with uncertain processing times, Computers \& Operations Research, Vol. 34, No. 10, 3055-3068, doi:10.1016/j.cor.2005.11.014

[7] Yu, G. D., Yang, Y., Zhao, X., Li, G. (2014). Multi-objective rescheduling model for product collaborative design considering disturbance, International Journal of Simulation Modelling, Vol. 13, No. 4, 472-484, doi:10.2507/IJSIMM13(4)CO17

[8] Song, L. J.; Gu, H. P.; Jin, S. Y.; Zhao, H. (2015). Rescheduling methods for manufacturing firms applying make-to-order strategy, International Journal of Simulation Modelling, Vol. 14, No. 4, 719-731, doi:10.2507/IJSIMM14(4)CO18

[9] Jiang, P.; Ding, J. L.; Guo, Y. (2018). Application and dynamic simulation of improved genetic algorithm in production workshop scheduling, International Journal of Simulation Modelling, Vol. 17, No. 1, 159-169, doi:10.2507/IJSIMM17(1)CO3

[10] Ribas, I.; Leisten, R.; Framinan, J. M. (2010). Review and classification of hybrid flowshop scheduling problems from a production system and a solutions procedure perspective, Computers \& Operations Research, Vol. 37, No. 8, 1439-1454, doi:10.1016/j.cor.2009.11.001

[11] Ruiz, R.; Vazquez-Rodriguez, J. A. (2010). The hybrid flow shop scheduling problem, European Journal of Operational Research, Vol. 205, No. 1, 1-18, doi:10.1016/j.ejor.2009.09.024

[12] Bang, J.-Y.; Kim, Y.-D. (2011). Scheduling algorithms for a semiconductor probing facility, Computers \& Operations Research, Vol. 38, No. 3, 666-673, doi:10.1016/j.cor.2010.08.010

[13] Joo, B. J.; Choi, Y. C.; Xirouchakis, P. (2013). Dispatching rule-based algorithms for a dynamic flexible flowshop scheduling problem with time-dependent process defect rate and quality feedback, Procedia CIRP, Vol. 7, 163-168, doi:10.1016/j.procir.2013.05.028

[14] Kianfar, K.; Ghomi, S. M. T. F.; Karimi, B. (2009). New dispatching rules to minimize rejection and tardiness costs in a dynamic flexible flow shop, International Journal of Advanced Manufacturing Technology, Vol. 45, No. 7-8, 759-771, doi:10.1007/s00170-009-2015-x

[15] Kia, H. R.; Davoudpour, H.; Zandieh, M. (2010). Scheduling a dynamic flexible flow line with sequence-dependent setup times: a simulation analysis, International Journal of Production Research, Vol. 48, No. 14, 4019-4042, doi:10.1080/00207540902922869

[16] Lodree Jr., E.; Jang, W.; Klein, C. M. (2004). A new rule for minimizing the number of tardy jobs in dynamic flow shops, European Journal of Operational Research, Vol. 159, No. 1, 258263, doi:10.1016/S0377-2217(03)00404-1

[17] Gholami, M.; Zandieh, M.; Alem-Tabriz, A. (2009). Scheduling hybrid flowshop with sequence dependent setup times and machines with random breakdowns, International Journal of Advanced Manufacturing Technology, Vol. 42, No. 1-2, 189-201, doi:10.1007/s00170-008-1577$\underline{3}$

[18] Kuo, Y.; Yang, T.; Cho, C.; Tseng, Y.-C. (2008). Using simulation and multi-criteria methods to provide robust solutions to dispatching problems in a flow shop with multiple processors, 
Mathematics and Computers in Simulation, Vol. 78, No. 1, 40-56, doi:10.1016/ j.matcom.2007.06.002

[19] Lee, G.-C. (2014). Real-time order flowtime estimation methods for two-stage hybrid flowshops, Omega, Vol. 42, No. 1, 1-8, doi:10.1016/j.omega.2013.02.004

[20] Choi, H.-S.; Kim, J.-S.; Lee, D.-H. (2011). Real-time scheduling for reentrant hybrid flow shops: a decision tree based mechanism and its application to a TFT-LCD line, Expert Systems with Applications, Vol. 38, No. 4, 3514-3521, doi:10.1016/j.eswa.2010.08.139

[21] Sabuncuoglu, I.; Touhami, S. (2002). Simulation metamodeling with neural networks: an experimental investigation, International Journal of Production Research, Vol. 40, No. 11, 24832505, doi: $10.1080 / 00207540210135596$

[22] Araz, O. U.; Eski, O; Araz, C. (2008). Determining the parameters of dual-card Kanban system: an integrated multicriteria and artificial neural network methodology, International Journal of Advanced Manufacturing Technology, Vol. 38, No. 9-10, 965-977, doi:10.1007/s00170-007$\underline{1138-1}$

[23] Hachicha, W. (2011). A simulation metamodelling based neural networks for lot-sizing problem in MTO sector, International Journal of Simulation Modelling, Vol. 10, No. 4, 191-203, doi:10.2507/IJSIMM10(4)3.188

[24] Rathinam, B.; Govindan, K.; Neelakandan, B.; Raghavan, S. S. (2015). Rule based heuristic approach for minimizing total flow time in permutation flowshop scheduling, Technical Gazette, Vol. 22, No. 1, 25-32, doi:10.17559/TV-20130704132725

[25] Mamdani, E. H. (1974). Application of fuzzy algorithms for control of simple dynamic plant, Proceedings of the Institution of Electrical Engineers, Vol. 121, No. 12, 1585-1588, doi:10.1049/piee.1974.0328

[26] Sugeno, M. (1985). Industrial Applications of Fuzzy Control, Elsevier Science Inc., New York

[27] Dhimish, M.; Holmes, V.; Mehrdadi, B.; Dales, M. (2018). Comparing Mamdani Sugeno fuzzy logic and RBF ANN network for PV fault detection, Renewable Energy, Vol. 117, 257-274, doi:10.1016/j.renene.2017.10.066

[28] Opricovic, S. (1998). Multicriteria Optimization of Civil Engineering Systems, PhD Thesis, Faculty of Civil Engineering, Belgrade

[29] Duckstein, L.; Opricovic, S. (1980). Multiobjective optimization in river basin development, Water Resources Research, Vol. 16, No. 1, 14-20, doi:10.1029/WR016i001p00014

[30] Gul, M.; Celik, E.; Aydin, N.; Gumus, A. T.; Guneri, A. F. (2016). A state of the art literature review of VIKOR and its fuzzy extensions on applications, Applied Soft Computing, Vol. 46, 6089, doi:10.1016/j.asoc.2016.04.040

[31] Peace, G. S. (1993). Taguchi Methods: A Hands-On Approach, Addison-Wesley, Reading

[32] Kacker, R. G.; Lagergren, E. S.; Filliben, J. J. (1991). Taguchi's orthogonal arrays are classical designs of experiments, Journal of Research of the National Institute of Standards and Technology, Vol. 96, No. 5, 577-591, doi:10.6028/jres.096.034

[33] MathWorks. Matlab Toolbox, from https://ch.mathworks.com/help/fuzzy/mamdani-fuzzyinference-systems.html, accessed on 17-09-2017

[34] Cheng, T. C. E.; Gupta, M. C. (1989). Survey of scheduling research involving due date determination decisions, European Journal of Operational Research, Vol. 38, No. 2, 156-166, doi: 10.1016/0377-2217(89)90100-8

[35] Savsar, M.; Choueiki, M. H. (2000). A neural network procedure for Kanban allocation in JIT production control systems, International Journal of Production Research, Vol. 38, No. 14, 3247-3265, doi: $10.1080 / 002075400418243$ 